\title{
Uso de un videojuego educativo como herramienta para aprender historia del Perú
}

\section{The use of a video game as an educational tool to learn the history of Peru}

\author{
Inés Evaristo Chiyong \\ Ricardo Navarro Fernández \\ Vanessa Vega Velarde \\ Teresa Nakano Osores \\ Pontificia Universidad Católica del Perú (Perú)
}

\section{Resumen}

La enseñanza de la historia es una tarea compleja debido a los retos de aprendizaje en los estudiantes como el generar un razonamiento crítico y crear un sentimiento de patriotismo. Estos objetivos son difíciles de alcanzar ya que generalmente aprender Historia se asocia a un aprendizaje memorístico de información. Esta situación obliga a pensar en diferentes estrategias, especialmente el uso de nuevas tecnologías informáticas e interactivas como herramientas pedagógicas. Dentro de estas, los videojuegos han tenido bastante acogida en los estudiantes. De esta forma, el presente estudio tiene como objetivo identificar la influencia de un videojuego de estrategia en tiempo real en el aprendizaje de los estudiantes. Para dicho propósito se realizó una intervención a tres grupos de estudio formados por 561 estudiantes de educación secundaria de ocho colegios de la ciudad de Lima. Un grupo solo utilizaría un videojuego, otro grupo solo recibiría clase de historia, y un tercer grupo tendría ambos estímulos. Se utilizó una prueba de historia, antes y después de la intervención, para medir el rendimiento académico. Lo resultados obtenidos demostraron que el videojuego como complemento a las clases del docente tuvo un mayor efecto en las calificaciones de los estudiantes. Esto demostraría que los videojuegos de este tipo podrían ser utilizables como una herramienta pedagógica en la enseñanza de la Historia.

Palabras clave: educación; tecnologías de la información y de la comunicación; método educativo; información pedagógica; innovación pedagógica; nuevas tecnologías.

\begin{abstract}
Teaching history is a complex task due to the pedagogical challenges that teachers face, especially because the goal is to promote critical thinking and create a feeling of patriotism among students. However, this objective is hard to achieve because History learning is based on memorization. This situation calls for a look into new technologies such as video games, that serve as educational tools which may potentially help solve this problem. Thus, the present study aims to demonstrate the usefulness and effectiveness of a real time strategy (RTS) game based on historical events about Peruvian independence. In order to achieve this
\end{abstract}


goal, an intervention with three groups was conducted, consisting of 561 students from eight schools in Lima. One group used only the video game, another group was taught only by a teacher, and a third group was exposed to both stimuli. Academic performance of students on the subject with a test input and output was measured. The results showed that the use of the video game, in addition to history classes, had a better impact on student grades and performance. This would suggest that this type of game could be used as a pedagogical tool in History teaching.

Keywords: education, information and communication technologies; educational method; pedagogical information; pedagogical innovation; new technologies.

Un objetivo importante de la enseñanza de la historia es construir conocimientos y actitudes relacionadas a la identidad de la persona con el país donde nació, así como el desarrollo de procesos cognitivos complejos vinculados al aprendizaje de estos contenidos. Esto implica que los estudiantes deben analizar y comprender distintos hechos históricos -nacionales e internacionales- para poder realizar juicios de valor hacia ellos y, de manera extendida, a la realidad actual de su país y del mundo (Valverde, 2010). Sin embargo, existen dificultades que pueden obstaculizar la enseñanza de la asignatura de historia. Una de las dificultades más comunes es el uso del aprendizaje memorístico de los hechos históricos.

El aprendizaje memorístico es una práctica común en la enseñanza en general; sin embargo, en la asignatura de Historia, puede ser perjudicial para el aprendizaje de los estudiantes, debido a que no se incita al razonamiento ni al análisis de lo ocurrido (Valverde, 2010). Asimismo, es importante resaltar que el aprendizaje memorístico no es necesariamente fomentado por los docentes, sino que los estudiantes asumen dicha postura como la más adecuada para aprender este tipo de contenidos. A esta realidad se le suma el uso de herramientas y técnicas educativas que refuerzan el aprendizaje memorístico (Sáiz y Colomer, 2014). Esto implica la repetición literal de información, listar una gran cantidad de datos y hechos de los textos o materiales que utilizan, poco espacio para la reflexión, la crítica y la creación, entre otros (Sáiz y Colomer, 2014).

Esta problemática se ha abordado a partir de distintas iniciativas que tenían el objetivo de mejorar esta situación. Una de ellas es implementar software interactivos y lúdicos (videojuegos) como herramienta pedagógica para apoyar al docente en el proceso de enseñanza-aprendizaje de dicha asignatura. El presente estudio tiene como objetivo implementar una herramienta educativa (videojuego) diseñada por el Grupo AVATAR PUCP para ser utilizada como un material educativo para una clase de Historia. El videojuego se centra en la rebelión en el Cuzco, liderada por Mateo Pumacahua y los hermanos Angulo, en el año 1814. 


\section{LA ENSEÑANZA DE LA HISTORIA EN EL CONTEXTO EDUCATIVO PERUANO}

La enseñanza de Historia en el colegio tiene dos objetivos (Carretero y Montanero, 2008): (1) Construir identidad en los estudiantes; (2) Construir conocimientos sobre los hechos históricos.

El primer objetivo hace referencia a la generación de un sentimiento patriótico que busca construir la identidad del ciudadano como miembro activo de su país. Para ello, en las clases se utiliza temas relevantes que enaltecen a personajes históricos (héroes de la patria), así como resaltar la importancia de los hechos históricos para un país y sus ciudadanos (Pagès, 2009; Carretero et al., 2008). El segundo objetivo se centra en desarrollar el razonamiento lógico de los estudiantes al analizar hechos históricos. Para ello, se busca que el estudiante identifique la secuencia, causas y consecuencias, para que así el estudiante pueda ejercitar y desarrollar habilidades cognitivas complejas (Pagès, 2009; Carretero et al., 2008).

En ese sentido, el Diseño Curricular Nacional del Perú (MINEDU, 2009) toma en cuenta ambos objetivos y señala características pedagógicas que deben cumplirse para la enseñanza de la asignatura de Historia. Una de estas -considerada una de las más importantes- es el carácter vivencial que debe tener la clase, ya que es importante que una clase permita que el estudiante se sienta inmerso en la experiencia (Gros, 2009). Para el logro de estos propósitos, la metodología de enseñanza debe generar un contexto que permita acercar a los estudiantes a los hechos de manera visual, espacial y temporal (Gros, 2009). En este sentido, lo que se propone es que el aprendizaje no sea solamente teórico, sino que se utilice materiales y actividades que permitan ejemplificar los conceptos teóricos. Esto permite construir conceptos relevantes para formar una conciencia acerca de uno mismo en relación con el contexto cívico, político y cultural (MINEDU, 2009). Asimismo, permite que los estudiantes comprendan y asuman un rol protagónico y autónomo, dirigido a ser parte del cambio, de la continuidad y causalidad de la historia (Chávez, 2006; Moreno, 2010; Sallés, 2010; Valle, 2011).

Un primer paso para enseñar historia es contextualizar a los estudiantes sobre los sucesos y personajes más importantes del período de tiempo que se quiere abarcar (Valle, 2011). Se debe ubicar los hechos en un espacio temporal y se debe lograr que el estudiante pueda tener una postura crítica hacia los temas trabajados en clase. Lograr esto no resulta una tarea sencilla para los docentes.

Como se mencionó anteriormente, en el Perú predomina un aprendizaje memorístico, pasivo y limitado de la historia (Chávez, 2006; Valle, 2011) que impide la construcción de conocimientos -no solo de fechas y hechos específicos, sino también de sentimientos patrióticos- que cumplan con los objetivos de aprendizaje trazados para los estudiantes (Gros, 2009). Esta situación se complica con la inadecuada capacitación que tienen los docentes para enseñar cursos de temáticas más especializadas (como lo es historia, matemáticas o química), lo que conlleva 
a que los estudiantes no reciban una formación adecuada (López, 2003). En ese sentido, el docente peruano se basa en los lineamientos temáticos propuestos por el DCN, en donde selecciona episodios históricos para elaborar su clase (Carretero, Rosa, González, 2006) que consta de dos o tres horas pedagógicas a la semana, que en muchos casos no es suficiente. Sin embargo, las carencias formativas y pedagógicas del docente actual, así como la escasez de materiales educativos -bibliotecas, laboratorios, material audiovisual y multimedia- funcionales para la enseñanza, dificultan reproducir o vivenciar un hecho histórico dentro del salón de clase u observar el pasado con las características que realmente tenía (Valverde, 2010). Estas deficiencias hacen que la historia se oriente al manejo de información sin significado para el estudiante (Valle, 2011).

Dado estos antecedentes, una alternativa que empieza a tener resultados positivos son los videojuegos utilizados como herramientas pedagógicas, ya sean estos videojuegos comerciales o educativos. Tal como lo señalan estudios recientes, el uso de videojuegos como recurso pedagógico puede permitir que el aprendizaje sea vivencial y entretenido, así como fomentar y desarrollar habilidades cognitivas superiores (Carretero et al., 2008; Gerber y Scott, 2011; Watson, Mong y Harris, 2011; Connollya, Boylea, MacArthur, Haineya y Boyle, 2012; All, Castellar y Van Looy, 2014; Kiili, Devlin, Perttula, Tuomi y Lindstedt, 2015), ayudar a desarrollar la creatividad y aumentar la motivación de los estudiantes (Papastergiou, 2009; Jackson, Witt, Games, Fitzgerald, Von Eye y Zhao, 2011). Además, debido a su calidad gráfica, reglas y mecánicas, pueden cumplir con los requerimientos para promover un aprendizaje lúdico, inmersivo y participativo (Cuenca y Martín, 2010; Watson et al., 2011).

\section{EL APRENDIZAJE DE LA HISTORIA MEDIANTE EL USO DE VIDEOJUEGOS}

Los videojuegos que tienen como contexto y base argumentativa un momento histórico real, han sido de particular interés dado su potencial para ser utilizados como herramientas pedagógicas. Squire (citado en Carretero et al., 2008) realizó un estudio con el juego de estrategia Civilization III y sus resultados apuntaron a que los videojuegos de este tipo podrían ser utilizados como materiales educativos e influir en la construcción de nuevos conocimientos históricos, así como cambios en las posturas e ideas de los estudiantes.

De la misma manera, Gros y Garrido (2008) utilizaron el videojuego Age of Empires II para conocer cómo el videojuego cumplía con el proceso de enseñanzaaprendizaje en estudiantes que oscilaban entre los 12 y 14 años. Se observó que los estudiantes más experimentados en el juego ayudaban a aquellos que no demostraban tanto dominio en el mismo, manifestándose así una zona de desarrollo próximo (ZDP) entre estos estudiantes que permitía la existencia de un aprendizaje colaborativo (Gros et al., 2008). Ayén (2010) refiere que algunos estudiantes poseen 
una mayor conexión y conocimientos de historia a partir de los videojuegos que utilizan, ya que establecen una relación entre los sucesos que se enseñan y los juegos que incluyen referencias a dichos sucesos. Esto permite que el estudiante tenga una motivación previa por los conceptos y sucesos, lo que hace más sencillo la enseñanza de los mismos. Respecto al género de los videojuegos, Sedeño (2010) señala que los de estrategia en tiempo real son útiles dentro de la enseñanza de contenidos históricos, no solo a nivel de conocimiento, sino también para fomentar el interés de los estudiantes por los temas estudiados.

Pese a lo anteriormente expuesto, una de las problemáticas que se destaca en el uso de videojuegos comerciales es que, al no haber sido diseñados con el propósito de enseñar historia, no responden a un objetivo educativo en particular, por lo que los aprendizajes que los estudiantes pueden construir no necesariamente siguen una línea educativa establecida ni se relacionan con los temas trabajados en el salón de clase (Gros, 2009). Sin embargo, existe evidencia en un estudio que intentó compensar esta debilidad en los estudios con videojuegos. Watson, Mong y Harris (2011) utilizaron un videojuego dentro del salón de clase para enseñar a los alumnos hechos históricos ocurridos durante la Segunda Guerra Mundial. Este proyecto se articuló con los objetivos de aprendizaje de una clase y se diseñó para que los estudiantes pudieran utilizarlo durante tres días en una semana. A continuación del uso del videojuego se procedía a recolectar la información a partir de entrevistas, donde los estudiantes señalaron que el cambio del paradigma pedagógico utilizado en el salón de clase ayudó a que se sintieran más motivados en los conceptos estudiados. Esto, junto a una adecuada guía del docente, facilitó que los estudiantes construyeran conocimientos sobre el periodo histórico estudiado.

En la República Checa se desarrolló un videojuego cuyo objetivo es enseñar la historia del siglo 20 de dicho país (Šisler, Brom, Cuhra, Činátl y Gemrot, 2012). Los contenidos presentados en el juego se encuentran orientados a que el estudiante aprenda sobre los hechos históricos, políticos y sociales de su país. Además, SáezLópez, Miller, Vásquez-Cano y Domínguez-Garrido (2015) utilizaron el videojuego de Minecraft dentro del salón de clase para enseñar los distintos contenidos históricos sobre la arquitectura a través de la historia. Los resultados en este estudio fueron positivos, particularmente en la actitud de los estudiantes a aprender sobre conceptos que no son particularmente agradables para ellos.

Sobre la base de estas experiencias y reflexiones, se podría plantear la premisa que un videojuego diseñado con un objetivo educativo podría ser utilizado como una herramienta pedagógica complementaria para el aprendizaje de la historia. Por ello, el objetivo del presente estudio es comprobar si el uso de un videojuego educativo -sobre un evento de la historia peruana- puede ser utilizado como una herramienta pedagógica que ayude al docente. Asimismo, identificar si mejora el desempeño académico y la motivación de los estudiantes para seguir aprendiendo sobre el tema presentado. En el presente estudio, se consideran variables de análisis como el tipo de institución educativa (privada o pública), la experiencia previa en el uso 
de videojuegos, la preferencia en el tipo de videojuegos y el sexo de los estudiantes. Se propone un diseño cuasi experimental con un grupo experimental y dos grupos de control.

\section{MÉTODO}

\section{Participantes}

La muestra del presente estudio estuvo conformada por 561 estudiantes de $3^{\circ}$ grado de secundaria, pertenecientes a ocho colegios públicos y privados de Lima Metropolitana ${ }^{1}$. Los estudiantes provenían de colegios de un nivel socioeconómico medio. El 80.4\% de los estudiantes asistió a todas las sesiones, mientras que $19.3 \%$ de los estudiantes no asistió a todas las sesiones y $0.4 \%$ no asistió a ninguna de ellas.

La distribución de los participantes según sexo fue: 269 varones (48.9\%) y 281 mujeres (51.1\%). Además, el 80\% de los participantes tienen entre 14 y 15 años (Media: 14.04, Desviación Estándar: .681). El 22\% de la muestra pertenecía a un colegio público, mientras que $78 \%$ pertenecía a un colegio privado.

Más de un tercio de la muestra afirma que juega videojuegos (77\%), sobre todo de estrategia (31.2\%) y combate (15.2\%), a los cuales suelen acceder los fines de semana, entre una y dos horas (41.7\%), desde sus casas (82\%). Finalmente, el $34 \%$ de la muestra considera que tiene un nivel intermedio de dominio en temas de videojuegos y el $44 \%$ considera que tiene un nivel de mayor experticia empleando videojuegos.

\section{Instrumentos y materiales}

Para el presente estudio se utilizaron los siguientes instrumentos y materiales:

- Ficha de datos: Elaborada con el objetivo de recoger información acerca de las características demográficas de los participantes y sus hábitos con respecto al uso de los videojuegos (horas y días de juegos, lugar donde juegan, tipo de juego, percepción sobre el dominio en videojuegos y con quien juega).

- Prueba de conocimientos: Este instrumento permite identificar el nivel de conocimiento y la apreciación de la importancia de los estudiantes respecto al tema de la Rebelión de Cusco en 1814, que lideró Mateo Pumacahua junto a los hermanos Angulo. Su elaboración contó con la participación de un historiador, además del apoyo de los investigadores del estudio. El cuestionario consta de dieciséis reactivos: quince con preguntas cerradas y una pregunta abierta. Cabe señalar que el estilo de preguntas fueron similares a lo que los estudiantes responden en sus evaluaciones en el colegio (preguntas dicotómicas, de verdadero y falso, ordenar, selección múltiple). 
- Cuestionario de motivación: el instrumento consta de 10 preguntas que permiten recoger la motivación de los estudiantes por los temas históricos tratados en el videojuego. Este instrumento fue elaborado por los investigadores y tuvo un nivel de confiabilidad de 0.86 .

- Videojuego "1814: La Rebelión del Cusco". Software educativo desarrollado por el Grupo AVATAR PUCP, grupo de investigación de tecnologías educativas. Combina la complejidad de un juego de rol y la acción de un juego de estrategia en tiempo real. En este videojuego se recrean los principales escenarios de la revuelta ocurrida en el año 1814 en el Perú. Se compone de tres partes diferenciadas: cinemáticas con información del contexto, diapositivas con información histórica y el juego propiamente dicho, que se divide en cinco misiones, las cuales pueden ser resueltas en un mínimo de cuatro horas. Para ganar en el juego, el jugador debe completar todos los niveles en que se presentan puntos importantes de la historia. En primer lugar, la historia del juego inicia con la Toma de la Audiencia de la ciudad del Cusco, en donde se lucha contra el Gobernador Pardo.

- Después, el jugador tiene que reclutar soldados, conseguir provisiones para el ejército patriota y buscar a Mateo Pumacahua para que se sume a la rebelión. Luego, se libra la batalla de la Apacheta, en la que el jugador tiene que buscar y derrotar al intendente José Gabriel Moscoso y al mariscal Francisco Picoaga, ambos realistas.

- Finalmente, el videojuego termina con la batalla de Umachiri, la cual constituyó el punto culminante de la revolución de Mateo Pumacahua.

\section{Procedimiento}

Para el presente estudio se seleccionaron ocho colegios de manera intencional (Hernández, Fernández y Baptista, 2010) que cumplieran con dos características: a) tener proyectos institucionales referidos a la incorporación de las TIC en el proceso educativo; b) laboratorio de cómputo disponible para poder llevar a cabo la intervención. Las instituciones educativas fueron invitadas a participar del estudio mediante una carta enviada por los investigadores.

El grupo de estudiantes proviene de tres tipos de instituciones educativas:

- Un colegio público perteneciente a la red de colegios de la Dirección General de Tecnologías Educativas (DIGETE) del Ministerio de Educación.

- Cuatro colegios pertenecientes a una red de colegios que tiene convenio en tecnologías educativas con la universidad que realiza el estudio.

- Tres colegios pertenecientes a un consorcio privado. 
Dentro de cada colegio, se asignaron de manera aleatoria los salones de acuerdo a los siguientes tres grupos de intervención:

- Grupo Experimental: Los estudiantes recibieron dos horas de clase dictadas por el docente de historia sobre la temática de la rebelión de Mateo Pumacahua y los hermanos Angulo. Adicionalmente, se aplicó el videojuego a los estudiantes durante cuatro horas pedagógicas y se les entregó el videojuego a los alumnos, con lo cual podían jugarlo el número de horas que desearan ${ }^{2}$.

- Grupo Control 1: Los estudiantes recibieron dos horas de clase dictadas por el docente de historia del aula, sobre la temática de la rebelión de Mateo Pumacahua y los hermanos Angulo.

- Grupo Control 2: Los estudiantes emplearon el videojuego en el laboratorio del colegio durante cuatro horas pedagógicas, bajo la supervisión de los evaluadores. Además, los estudiantes contaron con el videojuego, por lo que podían jugar el número de horas que desearan en otros espacios. Estos alumnos no recibieron clases sobre el tema.

Los grupos se distribuyeron tal como se muestra en la tabla 1.

Tabla 1. Distribución de estudiantes en grupos de estudio

\begin{tabular}{lcc}
\hline & Frecuencia & Porcentaje \\
\hline Grupo Experimental & 196 & 34.9 \\
Grupo Control 1 & 197 & 35.1 \\
Grupo Control 2 & 168 & 30.0 \\
\hline
\end{tabular}

La recolección de la información se realizó en dos etapas. En la primera, se aplicó una prueba de entrada a todos los participantes de los tres grupos de estudio antes de iniciar la intervención. Esta prueba tuvo como finalidad conocer el nivel de conocimientos que tenían respecto a los temas que se iban a desarrollar sobre la independencia del Perú en el año 1814.

Durante la intervención, los grupos que tenían la aplicación del videojuego fueron organizados con el apoyo del docente para que los estudiantes asistieran al laboratorio de cómputo por lo menos 4 horas pedagógicas. Cada estudiante tenía una computadora personal, pero eran libres de poder compartir información con sus compañeros. Se registraron las horas de juego y estas fueron supervisadas por los investigadores. No hubo participación del docente, también se registraron las dificultades y errores que se observaban en el videojuego. 
Los grupos que pasaron por clases estuvieron dirigidos por el mismo docente de historia de cada colegio. Los investigadores no observaron el desarrollo de estas clases.

Al finalizar las actividades de la intervención, se aplicó a los estudiantes la evaluación de salida, la cual incluyó las mismas preguntas que la evaluación de entrada. Además, se registraron dos preguntas adicionales en el grupo experimental y el grupo de control 2, que medía el número de horas que habían usado el videojuego fuera de la clase y el nivel al que habían llegado en el videojuego.

La prueba de entrada y la prueba de salida fueron tomadas en forma colectiva en cada sesión durante una hora. En cada una de las aplicaciones de los instrumentos de recolección de datos, las evaluadoras leyeron en voz alta las instrucciones y preguntaron si había alguna duda. Se les recalcó que las respuestas eran totalmente confidenciales y que no era una evaluación calificada; es decir, que no afectaría en su promedio escolar.

\section{Análisis de la Información}

Para el análisis de datos cuantitativos, se hizo uso del programa estadístico SPSS 21. Se realizó un análisis de consistencia, el cual conllevó a depuraciones de casos que no habían completado las diferentes etapas de la intervención.

Se compararon los puntajes de la prueba de entrada al inicio de la intervención en los tres grupos con el objetivo de saber si había diferencias significativas respecto al conocimiento previo del tema tratado. Igualmente, se compararon los puntajes de la prueba de salida en los tres grupos. Para poder comprobar la hipótesis de que el uso de un videojuego acompañado a las clases mejora el desempeño de los estudiantes en la prueba, se comparó las diferencias entre las dos pruebas dentro de cada grupo. Por último, se realizaron pruebas de correlaciones entre los puntajes hallados y los datos de la ficha de control y el puntaje de motivación para aprender historia.

\section{RESULTADOS}

Los resultados muestran que los grupos no tuvieron un mismo nivel de desempeño en la prueba de conocimientos al inicio de la intervención, $F(2,516)=11.125, p=.000$, siendo el grupo de control 1 el que mayor puntaje obtuvo en la prueba de entrada en comparación con los otros dos grupos de estudio. Es importante mencionar que no se encontraron diferencias significativas entre los puntajes del grupo experimental y el grupo de control 2 al comienzo del estudio $(\mathrm{p}=.315)$ (tabla 2). 
Tabla 2. Promedio del puntaje obtenido en la prueba de entrada

\begin{tabular}{lccccc}
\hline Grupo de estudio & $N^{*}$ & $M$ & $D E$ & \multicolumn{2}{c}{ Intervalo de confianza } \\
\cline { 4 - 6 } & & & & $\begin{array}{c}\text { Límite } \\
\text { Inferior }\end{array}$ & $\begin{array}{c}\text { Límite } \\
\text { superior }\end{array}$ \\
\hline Grupo & 192 & 6.72 & 5.45 & 5.91 & 7.47 \\
Experimental & 183 & 9.21 & 5.36 & 8.46 & 9.90 \\
Grupo Control 1 & 144 & 7.17 & 5.26 & 6.31 & 8.08 \\
Grupo Control 2 & 144 & & & & \\
\hline
\end{tabular}

${ }^{*} N$ : Número de participantes, M: Media; DE: Desviación Estándar

Al término de la intervención, los tres grupos incrementaron de manera significativa sus conocimientos sobre la rebelión de los hermanos Angulo y Mateo Pumacahua, $F(2,536)=3.734, p<.05$ (tabla 3$)$.

Tabla 3. Promedio del puntaje obtenido en la prueba de salida

\begin{tabular}{lccccc}
\hline \multirow{2}{*}{ Grupo de estudio } & $N^{*}$ & $M$ & $D E$ & \multicolumn{2}{c}{ Intervalo de confianza } \\
\cline { 4 - 6 } & & & & $\begin{array}{c}\text { Límite } \\
\text { Inferior }\end{array}$ & $\begin{array}{c}\text { Límite } \\
\text { superior }\end{array}$ \\
\hline Grupo Experimental & 189 & 13.4 & 4.81 & 12.75 & 14.07 \\
Grupo de control 1 & 187 & 12.93 & 4.27 & 12.36 & 13.50 \\
Grupo de control 2 & 163 & 12.07 & 4.68 & 11.33 & 12.77 \\
\hline
\end{tabular}

*N: Número de participantes, M: Media; DE: Desviación Estándar

Sin embargo, para poder identificar cuál de los grupos tuvo un mejor desempeño en la prueba, se comparó las diferencias de los puntajes de la prueba de entrada y la de salida, en cada uno de los grupos. Esto quiere decir que se comparó la variación que hubo entre la prueba de entrada y salida. Los resultados muestran que hay una diferencia significativa entre los tres grupos de estudio $F(2,497)=12.460, p=.000$. De esta forma, se pudo comprobar que el grupo experimental -es decir, aquellos que tuvieron clases de historia, así como horas de juego- tuvo mejores resultados que el grupo control 1 y el grupo control $2(\mathrm{p}<.05)$. Asimismo, el grupo que fue intervenido solo con horas de videojuegos tuvo un mejor desempeño en la prueba que el que llevó solo clase $(\mathrm{p}<.05)$. Es decir, aquellos grupos en donde se utilizó el videojuego como herramienta educativa mejoraron su rendimiento significativamente más que en los 
que no se hizo uso de este, con lo cual se comprueban las hipótesis de la investigación (tabla 4).

Tabla 4. Resultados del contraste de las diferencias entre la prueba de entrada y la prueba de salida, por grupo de estudio

\begin{tabular}{lccccc}
\hline Grupo de estudio & $N^{*}$ & $M$ & $D E$ & \multicolumn{2}{c}{ Intervalo de confianza } \\
\cline { 5 - 6 } & & & & $\begin{array}{c}\text { Límite } \\
\text { inferior }\end{array}$ & $\begin{array}{c}\text { Límite } \\
\text { superior }\end{array}$ \\
\hline Grupo Experimental & 185 & 6.76 & 5.91 & 6.33 & 7.20 \\
Grupo de control 1 & 174 & 3.78 & 5.60 & 3.35 & 4.21 \\
Grupo de control 2 & 141 & 4.87 & 5.68 & 4.39 & 5.35 \\
\hline
\end{tabular}

*N: Número de participantes, M: Media; DE: Desviación Estándar

Finalmente, se realizó una correlación entre la diferencia de rendimiento entre la prueba de entrada y salida y las variables como sexo, grado de estudio, horas de uso de videojuegos durante la semana, horas de uso de videojuegos los fines de semana, el total de horas de uso del videojuego "1814: La Rebelión del Cusco" fuera de la intervención y la motivación sobre el tema histórico. No se encontró ninguna correlación significativa, por lo que se podría afirmar que los efectos en el puntaje de los estudiantes se deben principalmente a la intervención (tabla 5).

Tabla 5. Relaciones entre las variables de control y la diferencia de las pruebas de entrada y salida

\begin{tabular}{|c|c|c|c|c|c|c|c|}
\hline & & Sexo & $\begin{array}{l}\text { Grado } \\
\text { de } \\
\text { estudio }\end{array}$ & $\begin{array}{c}\text { Horas de } \\
\text { juego de } \\
\text { lunes a } \\
\text { viernes }\end{array}$ & $\begin{array}{l}\text { Horas de } \\
\text { juego en } \\
\text { el fin de } \\
\text { semana }\end{array}$ & $\begin{array}{l}\text { Horas } \\
\text { jugadas }\end{array}$ & Motivación \\
\hline \multirow[t]{3}{*}{$\begin{array}{l}\text { Diferencia de } \\
\text { Rendimientos }\end{array}$} & $\begin{array}{l}\text { Correlación de } \\
\text { Pearson }\end{array}$ & -.03 & .08 & -.02 & -.02 & .02 & -.026 \\
\hline & $\mathrm{P}$ & .54 & .07 & .69 & .63 & .78 & .578 \\
\hline & $\mathrm{N}^{*}$ & 500 & 500 & 500 & 500 & 221 & 473 \\
\hline
\end{tabular}

${ }^{*} N$ : Número de participantes.

${ }^{*} N$ : Número de participantes. 


\section{DISCUSIÓN}

La presente investigación tuvo como objetivo demostrar si el desempeño académico de los estudiantes mejoraba al utilizar el videojuego educativo "1814: La Rebelión del Cusco" como material pedagógico en un salón de clase. Para ello, se contó con tres grupos de estudio: el grupo control 1 que solo llevó clases de historia, el grupo control 2 que solo jugó el videojuego, y el grupo experimental que fue expuesto tanto a las clases de historia como al videojuego. Se esperaba que los estudiantes que usaron el videojuego mejoraran su rendimiento en las pruebas de aprendizaje en comparación con el de estudiantes que no lo usaron.

Los resultados obtenidos señalan que en los tres tipos de intervención hubo mejoras significativas entre la prueba de entrada y salida. Esto era esperable debido a que la enseñanza de las rebeliones previas a la independencia del Perú (la rebelión de los hermanos Angulo y Mateo Pumacahua) no son temas que se desarrollan de forma consensuada en los colegios. Por ello, al ser un contenido nuevo, es esperable que los resultados de diferencias de rendimiento muestren incremento.

A pesar de esta mejora, cabe resaltar que los resultados demuestran que los grupos que incrementaron en mayor medida sus conocimientos sobre el tema, fueron los que emplearon el videojuego. Como primer punto, se encontró que el grupo de estudiantes que utilizó el videojuego, y a la vez tuvo clases sobre el tema de la rebelión en el Cusco, tuvo un mayor incremento en la puntuación de la prueba de salida en comparación con los demás grupos. Este es un hallazgo importante que indica que las tecnologías o software funcionan mucho mejor cuando se incorporan o se complementan con el trabajo del docente en aula. Este resultado elimina las posturas fatalistas con respecto a que las tecnologías reemplazarían al docente. Lo que obtuvimos fue que es mejor la integración de los dos aspectos: clases o intervención del docente y recurso (juego). Sabemos que se puede plantear que el factor tiempo puede haber sido una variable influyente, dado que el grupo experimental recibió "más horas" sobre el tema (clases más laboratorio con el videojuego); consideramos que más allá del tiempo, el tipo de intervención es lo que puede estar marcando la diferencia. La duración de la clase presencial fue planteada por el profesor en la medida que era el tiempo "adecuado" según su experticia en desarrollar dicho tema en aula. Pero sí es un dato a considerar para futuras investigaciones.

No obstante a este primer resultado (integración clases más videojuegos), queremos resaltar en este estudio un segundo punto de análisis. Se encontró que el grupo que empleó solo el videojuego, obtuvo una mejora significativa en comparación al grupo que solo llevó una clase tradicional sobre el tema. Los hallazgos concuerdan con las investigaciones encontradas acerca de cómo el uso de los videojuegos en clase pueden aumentar el rendimiento académico (Gros y Garrido, 2008; Carretero et al., 2008). Y también ponen en el debate las críticas sobre la forma en que los docentes están facilitando el proceso de aprendizaje y enseñanza de la historia, si realmente están logrando los objetivos propuestos en el área curricular para la 
educación secundaria. Estos resultados refuerzan lo encontrado por Evaristo y Vega (2013) donde los estudiantes manifiestan que el videojuego educativo les ayudó a comprender la historia (asignatura que consideran tediosa y aburrida) realizando una acción que consideraron divertida y entretenida como jugar. En dicho estudio la mayoría de los participantes mencionó que el videojuego podría usarse en la clase de historia, ya que explica el tema a tratar de manera clara y concisa, lo cual los ayuda a comprender y visualizar mejor la historia y, por ende, aumenta la motivación por aprender acerca del tema tratado.

Si bien se han realizado publicaciones acerca del uso de videojuegos en la enseñanza (Squire, citado en Carretero, 2008; Cuenca et al., 2010; Echeverría et al., 2011), no han sido comunes los estudios donde han sido utilizados como recurso pedagógico complementario a las clases. En este sentido, los resultados del presente estudio evidencian la potencialidad de los videojuegos educativos debido a que exponen a los estudiantes a estímulos audiovisuales que permiten capturar elementos propios de la época (arquitectura, vestimenta, roles sociales, personajes). Asimismo, el aprendizaje de datos históricos se vuelve relevante para lograr con éxito las misiones que propone el juego, cobrando un sentido al aprendizaje de los mismos. Esto último se ve optimizado por la experiencia directa que ofrece el juego, es decir, el estudiante vivencia la historia a partir de sus propias acciones y de la información con la que cuenta. Todo ello genera una forma diferente de aprender los hechos históricos para favorecer, a partir de ellos, el análisis crítico, construcción del tiempo histórico y los aspectos afectivos asociados a esta área curricular.

Por su parte, Cuenca (2006) afirma que los videojuegos facilitan la comprensión de los referentes culturales que son difíciles de entender debido a que los estudiantes viven en una época y un contexto diferente. El valor de los videojuegos, como sistemas complejos interactivos ayuda a cubrir las limitaciones que tienen los materiales que los docentes disponen para enseñar historia, muchas veces descontextualizados y con un enfoque en el aprendizaje memorístico (Valle, 2011).

En esta misma línea, diferentes autores resaltan la capacidad de los videojuegos para presentar información de manera interactiva y lúdica, lo cual contribuye a que el estudiante pueda participar activamente de su aprendizaje y dirigir la atención en el análisis de las situaciones presentadas. Esto permite que, basados en los contenidos del juego, el estudiante pueda desarrollar su pensamiento crítico (Sahhuseyinoglu, 2007; Gee, 2004; Prensky, 2006). Por su parte, Gros (2009) señala el efecto de los videojuegos educativos, que tienen un objetivo de aprendizaje definido, en la asimilación de contenidos nuevos. Tal como se ha mostrado con el aprendizaje en torno a hechos y personajes logrado por los estudiantes que tuvieron acceso a jugar el videojuego "1814: La Rebelión del Cusco". Estas ventajas también son percibidas por los participantes cuando expresan que el videojuego les permitió "aprender de una forma más dinámica, fácil y entretenida” (Evaristo y Vega, 2013).

Con respecto a variables asociadas a los aprendizajes logrados a partir del videojuego "1814: La Rebelión del Cusco", los resultados han demostrado que este 
efecto (incremento en el aprendizaje) no ha sido influenciado por otras variables como la motivación, la edad, tipo de colegio o el sexo del estudiante, sino que se atribuyen directamente al tipo de intervención.

Entonces, ¿qué hace que un videojuego pueda promover el aprendizaje de la historia? Como señalan diferentes autores y el presente estudio ha evidenciado, el videojuego posee características inherentes que lo hace llamativo para los adolescentes. En primer lugar, un videojuego es un sistema organizado de reglas, procedimientos y metas que requiere la participación activa del jugador, lo compromete con el logro de las misiones u objetivos. Además de las características audiovisuales, cuenta con una historia que traslada al participante a un espacio virtual que lo aproxima a aspectos culturales y sociales de un hecho histórico que le permite identificar y comprender los hechos históricos de manera lúdica (Gramigna y Gonzáles-Faraco, 2009; Cuenca et al., 2010). Ello responde a la necesidad de contextualizar el pasado que requiere el aprendizaje de la historia (Carretero et al., 2008), lo que permite que sea interactivo y que el estudiante se sienta inmerso en la situación que se quiere enseñar. Este tipo de material educativo, siendo utilizado pedagógicamente dentro del salón de clase, permite que el estudiante pueda conocer de manera más realista y lúdica los hechos históricos que se quiere enseñar. El videojuego de estrategia "1814: La Rebelión del Cusco" ha logrado satisfacer esa necesidad. Asimismo, este estudio permite aportar evidencias que apoyan el uso de los videojuegos con temáticas históricas de tipo estrategia como herramientas para la enseñanza del área curricular de historia, generando un efecto positivo en el aprendizaje que no se logró con otro tipo de metodología que el docente utilizó en su clase.

\section{CONCLUSION}

El presente estudio permite argumentar, así como varios estudios similares sobre el uso de videojuegos en un contexto educativo, el beneficio que conlleva el uso adecuado y estructurado de un videojuego dentro del salón de clase, como un material de apoyo a la clase del docente. Sabemos que, como toda investigación con recursos limitados, no podemos generalizar estos resultados. Este fue un primer intento en nuestro país de impulsar la investigación de uso de videojuegos con fines educativos. Pero somos conscientes que la medición de los aprendizajes es un tema complejo y es necesario para futuras investigaciones utilizar diferentes técnicas de evaluación que se vienen empleando tanto desde entrevistas a profundidad, observaciones, medición de respuestas cerebrales, fisiológica a partir del uso del juego, etc. Es nuestro principal reto: mejorar instrumentos para aproximarnos mejor a nuestra variable de estudio.

Por otra parte, como recomendaciones para futuras investigaciones, además de la mejora en los instrumentos de evaluación, consideramos que permitirá mostrar un contexto más complejo si se analiza qué tipos de conocimientos fueron los 
más influenciados por el uso del videojuego, ya que identificar qué características particulares del videojuego tienen ese efecto, permitiría expandir el campo de investigación. Asimismo, sería importante identificar qué aspectos del videojuego motivan a los estudiantes a seguir aprendiendo de los temas que se presentan en los videojuegos. Es importante añadir que, si bien el videojuego acompañado con la clase del docente es el que ha generado mayor efecto en el aprendizaje, fuera del contexto de investigación es el propio docente el encargado de promover estas innovaciones, por lo que es interesante investigar cómo se dan los comportamientos cuando es el docente quien orienta las sesiones de uso de los videojuegos.

Se puede concluir que el uso del videojuego "1814: La Rebelión del Cusco", como herramienta pedagógica complementaria a las clases de los docentes, ayuda a mejorar el desempeño de los estudiantes sobre el tema de la rebelión de los hermanos Angulo y Mateo Pumacahua. Este estudio es un aporte a la iniciativa de utilizar videojuegos dentro del contexto educativo, demostrar que los videojuegos no solo entretienen, sino que también motivan o apoyan a procesos cognitivos como memoria, discriminación, análisis etc.; el aporte principal de esta investigación estaba orientada en demostrar que realmente produce aprendizajes, aprendizajes que van en sinergia con lo que el docente pide a sus estudiantes como logro de aprendizaje. Es un aporte, en especial al sistema educativo peruano y a diferentes sistemas educativos aún incipientes en el uso de este tipo de recursos educativos. Es también un pequeño aporte para la tarea de la desmitificación de la imagen negativa que pueden tener los videojuegos en los padres de familia y docentes.

\section{NOTAS}

1. Uno de los tipos de colegio no contaba con promociones de $3^{\circ}$ de secundaria se consideró a los alumnos de $2^{\circ}$ de Educación secundaria.

2. Cabe señalar que los docentes del grupo experimental y control 1 desarrollaron su clase sobre "1814: La Rebelión del Cusco" después de haber pasado -semanas antespor una capacitación sobre la rebelión de los hermanos Angulo y Mateo Pumacahua realizada por un experto en historia del Perú. Luego de esta capacitación, los docentes desarrollaron, según su estilo, la sesión de aprendizaje.

\section{REFERENCIAS BIBLIOGRAFICAS}

Ayén, F. (2010). Aprender Historia con el juego Age of Empires. (Reseña). Proyecto Clio 36. ISSN: 1139-6237. Recuperado de: http://clio.rediris.es

All, A., Castellar, E. P. N., y Van Looy, J. (2014). Measuring Effectiveness in Digital Game-Based Learning: A Methodological
Review. International Journal of Serious Games, 1(2).

Carretero, M., Rosa, A., y González, M. (Eds.) (2006). Enseñanza de la historia y memoria colectiva. Buenos Aires: Paidós.

Carretero, M., y Montanero, M. (2008). Enseñanza y aprendizaje de la historia: 
aspectos cognitivos y culturales. Cultura $y$ Educación, 20(2), 133-142.

Chávez, T. (2006). La enseñanza de la historia del Perú en la educación secundaria durante la segunda mitad del siglo $X X$. Pontificia Universidad Católica del Perú: Lima.

Connollya, T., Boylea, E., MacArthur, E., Haineya, T., y Boyle, J. (2012). A systematic literature review of empirical evidence on computer games and serious games. Computers \& Education, 59, 661686.

Cuenca, J. (2006). La enseñanza de contenidos sociohistóricos y patrimoniales a través de los juegos informáticos de simulación. Treballs d'Arqueologia. Bellaterra: Servei de Publicacions de la Universitat Autónoma de Barcelona, 12, 111-126.

Cuenca, J., y Martín, M. (2010). Virtual games in social science education. Computers \& Education, 55, 1336-1345.

Echeverría, A., García-Campo, C., Nussbaum, M., Gil, F., Villalta, M., Améstica, M., y Echeverría, S. (2011). A framework for the design and integration of collaborative classroom games. Computers \& Education, 50, 1127-1136.

Evaristo, I., y Vega, M. (2013). "Con los videojuegos aprendo". Testimonios de estudiantes de secundaria sobre el uso de un videojuego educativo sobre historia del Perú. Publicación presentada en el II Congreso Internacional de Videojuegos y Educación. Recuperado de: https:/ dl.dropboxusercontent.com/u/4318784/ Libro CIVE 13.pdf

Gee, J. (2004). Lo que nos enseñan los videojuegos sobre el aprendizaje $y$ el alfabetismo. Málaga: Ediciones Aljibe

Gerber, S., y Scott, L. (2011). Gamers and gaming context: relationships to critical thinking. British Journal of Educational Technology, 42(5), 842-849.

Gramigna, A., y Gonzáles-Faraco, J., (2009). Videojugando se aprende: renovar la teoría del conocimiento y la educación. Comunicar 17(33), 157-164.

Gros, B., y Garrido, J. (2008). "Con el dedo en la pantalla": El uso de un videojuego de estrategia en la medición de aprendizajes curriculares. Teoría de la Educación: Educación y Cultura en la Sociedad de la Información, 9(3), 108-129.

Gros, B. (2009). Certezas e interrogantes acerca del uso de los videojuegos para el aprendizaje. Comunicación, 7(1), 251-264.

Hernández, R., Fernández, C., y Baptista, P. (2010). Metodología de la Investigación. México: McGraw Hill.

Jackson, L., Witt, E., Games, A., Fitzgerald, H., Von Eye, A., y Zhao, Y. (2011). Information technology use and creativity: findings from the children and technology project. Computers in Human Behavior. doi: 10.1016/j.chb.2011.10.006.

López, M. (2003). Bolivia, Paraguay y Perú. En I. Flores, (Ed.), ¿Cómo estamos formando a los maestros en América Latina? (103-118). Perú: Lima.

Kiili, K., Devlin, K., Perttula, T., Tuomi, P., y Lindstedt, A. (2015). Using video games to combine learning and assessment in mathematics education. International Journal of Serious Games, 2(4).

MINEDU (2009). Diseño Curricular Nacional de Educación Básica Regular. Ministerio de Educación: Lima, Perú.

Moreno, M. (2010). Aprender historia en ambientes virtuales. Tejuelo, 9, 58-82.

Pagés, J. (2009). Enseñar y aprender ciencias sociales en el siglo XXI: reflexiones casi al final de una década. Investigación en Educación, Pedagogía y Formación Docente, II Congreso Internacional. Libro 2, Medellín. Universidad Pedagógica Nacional, Universidad de Antioquia, Corporación Interuniversitaria de Servicios, (pp. 140-154).

Papastergiou, M. (2009). Digital game-based learning in high school computer science education. Computers \& Education, 52(1), 1-12. 
Prensky, M. (2006). Don't bother me mom - I'm learning. Estados Unidos: Paragon House.

Sáez-López, J. M., Miller, J., Vázquez-Cano, E., y Domínguez-Garrido, M. C. (2015). Exploring application, attitudes and integration of video games: MinecraftEdu in middle school. Journal of Educational Technology \& Society, 18(3), 114-128.

Saíz, J., y Colomer, J. (2014). ¿Se enseña pensamiento histórico en libros de texto de Educación Primaria? Análisis de actividades de historia para alumnos de 10-12 años de edad. History and History teaching, 40.

Sahhuseyinoglu, D. (2007). Educational games for developing critical thinking skills: pre-service english language teachers' views. Hacettepe Üniversitesi Eğitim Fakültesi Dergisi, 32, 266-273.

Sallés, N. (2010). La enseñanza de la historia a través del aprendizaje por descubrimiento: Evolución del proyecto treinta años después. Enseñanza de las Ciencias Sociales, 10, 3-10.
Sedeño, A. (2010). Videojuegos como dispositivos culturales: las competencias espaciales en educación. Comunicar, 17, 183-189.

Šisler, V., Brom, C., Cuhra, J., Činátl, K., y Gemrot, J. (2012). Stories from the History of Czechoslovakia, A Serious Game for Teaching History of the Czech Lands in the 2oth Century-Notes on Design Concepts and Design Process. In Entertainment Computing-ICEC 2012 (pp. 67-74).

Valle, A. (2011). El uso de las Fuentes escritas en la enseñanza de la historia. Análisis de textos escolares para tercero y cuarto de secundaria. Educar, 29(38), 81-106.

Valverde, J. (2010). Aprendizaje de la historia y simulación educativa. Tejuelo 9, 83-89.

Watson, W., Mong, C., y Harris, C. (2011). A case study of the in-class use of a video game for teaching high school history. Computers \& Education, 56, 466-474.

\section{PERFIL ACADÉMICO Y PROFESIONAL DE LOS AUTORES}

Inés Evaristo Chiyong. Master en Enseñanza y Aprendizaje Abiertos y a Distancia por la Universidad Nacional de Educación a Distancia, egresada de la Maestría en Políticas Educativas por Pontificia Universidad Católica del Perú (PUCP), Licenciada en Psicología Educacional por la PUCP. Ha dirigido proyectos de desarrollo e investigación relacionados al uso de las tecnologías interactivas como herramientas de mejora de la educación. Trabajó en proyectos de educación a distancia y en alternancia para la educación secundaria rural y urbana para el Ministerio de Educación del Perú. Fue coordinadora general del Grupo AVATAR de la PUCP, donde actualmente es investigadora principal. Actualmente es la Directora de Tecnologías para el Aprendizaje de la Universidad Tecnológica del Perú.

E-mail: ines.evaristochiyong@gmail.com

Ricardo Javier Navarro Fernández. Licenciado en Psicología Educacional por la Pontificia Universidad Católica del Perú. Es asistente de docencia en la especialidad de Psicología de la facultad de Letras y Ciencias Humanas de la PUCP. Se enfoca en estudios experimentales sobre el uso de nuevas tecnologías en la 
educación, así como en el diseñando de materiales educativos virtuales y cursos de e-learning.

E-mail: ricardo.navarro@pucp.pe

María Vanessa Vega Velarde. Bachiller en Psicología Educacional por la Pontificia Universidad Católica del Perú. Tiene experiencia en el diseño, desarrollo y evaluación de proyectos educativos con uso de tecnologías interactivas, especialmente videojuegos para mejorar el aprendizaje y motivación en estudiantes. Ha participado en el diseño de videojuegos para el aprendizaje de historia y comprensión de lectura en educación básica regular.

E-mail: vanessa.vega@pucp.pe

Teresa Nakano Osores. Licenciada en Psicología Educacional en la Pontificia Universidad Católica del Perú, Magister en Políticas Públicas y Sociales en la Universidad Pompeu Fabra (Barcelona) y Magister en Política y Gestión Universitaria en la Universidad de Barcelona. Amplia experiencia en el diseño y evaluación de proyectos educativos a nivel nacional para la educación superior. Asimismo, experiencia en el desarrollo de proyectos educativos con uso de tecnologías. Actual Directora Dirección de Servicios de Educación Técnica Productiva y Superior Tecnológica y Artística del Ministerio de Educación del Perú.

E-mail: tnakano@minedu.gob.pe

\section{DIRECCIÓN DE LOS AUTORES}

Grupo AVATAR PUCP

Pontificia Universidad Católica del Perú

Av. Universitaria 1801, San Miguel

Lima (Perú)

Fecha de recepción del artículo: 10/11/2015

Fecha de aceptación del artículo: 04/02/2016

\section{Como citar este artículo:}

Evaristo-Chiyong, I. S., Vega-Velarde, M. V., Navarro Fernandez, R., y Nakano Osores, T. (2016). Uso de un videojuego educativo como herramienta para aprender historia del Perú. RIED. Revista Iberoamericana de Educación a Distancia, 19(2), pp. 35-52. doi: http://dx.doi.org/10.5944/ried.19.2.15569 